\title{
Improved Face Shape Recovery and Re-illumination Using Convexity Constraints
}

\author{
Mario Castelán* and Edwin R. Hancock \\ Dept. of Computer Science, University of York, \\ York YO10 5DD, United Kingdom \\ \{mario, erh\}@cs.york.ac.uk
}

\begin{abstract}
This paper describes work aimed at developing a practical scheme for face analysis using shape-from-shading. Existing methods have a tendency to recover surfaces in which convex features such as the nose are imploded. This is a result of the fact that subtle changes in the elements of the field of surface normals can cause significant changes in the corresponding integrated surface. To overcome this problem, in this paper we describe a local-shape based method for imposing convexity constraints. We show how to modifying the orientations in the surface gradient field using critical points on the surface and local shape indicators. The method is applied to both surface height recovery and face re-illumination, resulting in a clear improvement.
\end{abstract}

\section{Introduction}

The problem of acquiring surface models of faces is an important one with potentially significant applications in biometrics, computer games and production graphics. Shape-from-shading is one of the most appealing methods, since this is a non-invasive process which mimics the capabilities of the human vision system.

In brief, SFS aims to solve the image irradiance equation, $E(x, y)=R(p, q, s)$, where $E$ is the intensity value of the pixel with position $(x, y), R$ is a function referred to as the reflectance map 6. The reflectance map uses the surface gradients $p=\frac{\partial Z(x, y)}{\partial x}$ and $q=\frac{\partial Z(x, y)}{\partial y}$ together with the light source direction vector $s$ to compute a brightness estimate which can be compared with the observed one using a measure of error. If the surface normal at the location $(x, y)$ is $n=(p, q,-1)$, then under Lambertian reflectance model, the image irradiance equation becomes $E(x, y)=n \cdot s$. In other words, the SFS problem is the one of recovering the surface that, after interaction with the environment (illumination conditions, reflectance properties of the object, inter-reflections) produces the radiances perceived by human eyes as intensities.

In general, though, SFS is an under-constrained problem since the two degrees of freedom for surface orientation (slant and tilt), must be recovered from

\footnotetext{
* Supported by National Council of Science and Technology (CONACYT), Mexico, under grant No. 141485.
} 
a single measured intensity value. Hence, it is frequently posed as that of minimizing cost functionals that capture constraints on the gradient field [6]. This is usually carried out through iterative schemes in the discrete domain. Despite sustained research activity in the field for some three decades, no SFS scheme has been demonstrated to work as accurately as the specially constrained SFS cases of photometric stereo 4 and statistical SFS[1]. The first of these methods requires at least three images of the same object illuminated from different viewpoints, while the second uses an aligned database of accurate surface information belonging to objects of the same class, i.e. faces.

When it comes to the original single-image problem, the restrictions imposed by most SFS schemes on the gradient field (smoothness, irradiance, integrability, unit length) are insufficient to overcome with these problems, generating errors that, if propagated iteratively, can result in miscalculations on the topography of the recovered surface. For instance, because of the concave-convex ambiguity, there may be regions where the sign of the curvature becomes reversed. As far as the problem of face reconstruction is concerned, for instance, the effect can be to cause high curvature features such as the nose to become imploded with respect to the remainder of the surface. As we will demonstrate in this paper, if such regions can be identified and the surface normal directions corrected, then the result is improved overall surface topography.

For face analysis, the use of SFS has proved to be an elusive task, since the concave-convex ambiguity can result in the inversion of important features such as the nose. To overcome this problem, domain specific constraints have been used. Dogvard and Basri 3] have exploited facial symmetry. Prados and Faugeras [8] have recently proposed a SFS scheme that, under the existence of a unique critical point (i.e. the brightest intensity appearing at only one location in the image) and a light source at the optical center of the camera (rather than a homogeneous horizontal light) gives qualitatively good facial reconstructions.

It is clear that a precise height map is difficult to obtain by integration of the field of surface normals delivered by SFS due to local errors in direction and potential reversal in the sign of the curvature. However, this does not imply that the entire field of surface normals is in error.

The outline of the this paper is as follows. First we review the concepts of local shape indicators. With this knowledge at hand we develop a method that can be used to reassign the surface gradient orientations. We provide experiments to evaluate the method on height recovery and re-illumination using single images of human faces. Finally, we present some conclusions and identify directions for future work.

\section{Local Shape Indicators}

Curvature attributes have been widely used in shape analysis, especially for segmentation and recognition. A local shape indicator is an scalar that conveys information concerning the local topography of a surface using its principal curvatures. The principal curvatures $\left(\kappa_{1}\right.$ and $\left.\kappa_{2}\right)$ are the eigenvectors of the Hessian 
matrix, which can be computed through local changes in the surface normal directions.

Local shape indicators are usually coupled. For instance, the $H K$ classification [2] uses the Gaussian and mean curvatures $H=\frac{\left(\kappa_{1}+\kappa_{2}\right)}{2}$ and $K=$ $\kappa_{1} * \kappa_{2}$ respectively. By distinguishing between the cases in which $\mathrm{H}$ and $\mathrm{K}$ are individually negative, zero or positive, it is possible on the basis of their joint behavior to assign topographic labels to points on a surface. A different and slightly more convenient set of attributes is the curvedness/shape-index representation developed by Koenderink and Van Doorn [7. Here the principal curvatures are used to compute the shape index $S=-\frac{2}{\pi} \arctan \left(\frac{\kappa_{1}+\kappa_{2}}{\kappa_{1}-\kappa_{2}}\right)$ for $\kappa_{1} \geq \kappa_{2}$; and the curvedness, $C=\left(\frac{\kappa_{1}^{2}+\kappa_{2}^{2}}{2}\right)^{1 / 2}$.

The shape index is an angular variable that relates directly to the local surface topography. It varies continuously from -1 to +1 as the surface changes through cup, rut, saddle-rut, saddle, saddle-ridge, ridge and dome, and cup again. The curvedness is simply the degree of curvature of the surface. The curvedness is a convenient indicator of potential surface discontinuity. The higher the curvedness, the more likely the presence of a rapid variation in height. For instance, in the case of faces, the curvedness is large for features such as the boundaries of the nose, mouth and eyes.

For our experiments, we utilize the local descriptors of shape-index and curvedness to characterize the regions on a gradient field where a change of orientation should be performed.

\section{Using Local Shape Indicators to Redirect SFS Gradient Fields}

Inevitably, any surface gradient field delivered by SFS will be inaccurate due to noise or albedo changes which cause variations in the intensities of the input image. SFS works well for objects that are uniformly concave or convex. However, if the object under study is more complex, with both concave and convex regions, then SFS can fail. In these situations although the recovered surface normal direction is consistent with the measured image brightness, the recovered surface does not reflect the structure of the object under study. In particular, there may be inversions of the sign of the surface curvature with convex regions appearing concave and vice-versa. However, in the case of faces (and many other objects) the surface under study is largely convex (with the exception of the eye-sockets, the bridge of the nose and areas around the lips). Based on this observation, we present a method for enforcing the convexity of the integrated surface while ensuring a global maximum on a particular position on it.

Formally stated, suppose $S$ is a smooth surface immersed in $\mathbb{R}^{3}$. Let $p$ and $U_{p}$ be a critical point of $S$ and a neighborhood of $p$ respectively. Suppose that $S$ is locally concave over $U_{p}$. Then, the new surface $\widetilde{S}$ constructed from $S$ by reversing the sign of all its partial derivatives, $S_{x}$ and $S_{y}$, is locally convex in $U_{p}$. Besides, a local maximum on $\widetilde{S}$ will be located at that point where the function ceases 

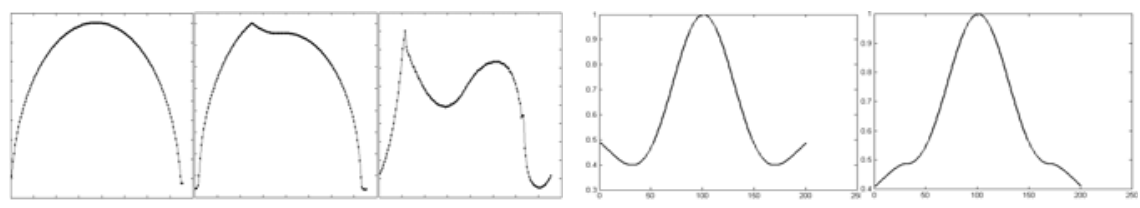

Fig. 1. Applying the method to a sphere and mexican hat(see text)

increasing and commences decreasing 1 . Suppose that all the partial derivatives of $\widetilde{S}$ with respect to $x, \widetilde{S}_{x}$, have negative sign before reaching the position of the critical point $p$ on the $x$ axis and have positive sign after reaching it, and that the same conditions hold for $\widetilde{S}_{y}$. The critical point $p$ on $U_{p}$ will be the position of the global maximum 2 of $\widetilde{S}$.

The basic idea underlying this paper is to enforce the condition that the integrated surface has a global height maximum at the tip of the nose. Such a point will serve as a division for the positive and negatively signed areas of the needle map. To enforce this condition we apply the simple rule:

$$
\begin{aligned}
& \breve{Z}_{x}(x, y)= \begin{cases}a b s\left(\hat{Z}_{x}(x, y)\right) & \text { if } x \leq a \text { and } \operatorname{ShI}(x, y) \geq \tau_{x} \\
-a b s\left(\hat{Z}_{x}(x, y)\right) & \text { if } x>a \text { and } \operatorname{ShI}(x, y) \geq \tau_{x} \\
\hat{Z}_{x}(x, y) & \text { otherwise }\end{cases} \\
& \breve{Z}_{y}(x, y)= \begin{cases}a b s\left(\hat{Z}_{y}(x, y)\right) & \text { if } y \leq b \text { and } \operatorname{ShI}(x, y) \geq \tau_{y} \\
-a b s\left(\hat{Z}_{y}(x, y)\right) & \text { if } y>b \text { and } \operatorname{Sh} I(x, y) \geq \tau_{y} \\
\hat{Z}_{y}(x, y) & \text { otherwise }\end{cases}
\end{aligned}
$$

where $\breve{Z}_{x}$ and $\breve{Z}_{y}$ are the updated gradients, $\hat{Z}_{x}$ and $\hat{Z}_{y}$ are the original gradients, $a$ and $b$ are the coordinates for the position of the desired global height maximum, on the $x$ and $y$ axis respectively. A local shape indicator $S h I$, which could be either the curvedness, the shape index or the mean and gaussian curvatures, is compared to the thresholds $\tau_{x}$ and $\tau_{y}$ for deciding whether the element of the gradient field at the location $(x, y)$ will be altered or not.

The following diagrams show the behavior of the method when applied to the derivatives of a sphere and a mexican hat. Both experiments were realized using the curvedness indicator.

To illustrate the global height maximum enforcement procedure, Figure 1 (left) shows the results of applying the method to the derivatives of a sphere with radius 75 units. From left to right, transversal plots of the recovered surface on the $x$ axis are shown. The peak coordinates $(a, b)$ are both set to 75,50 and 20 respectively, and $\tau_{x}$ and $\tau_{y}$ are set to zero: all the derivatives are taken into

${ }^{1}$ Of course, $\widetilde{S}$ will present many local maxima for a face-like surface.

${ }^{2}$ It might be a maximum or a minimum depending on the integration method. 
account to expose the extreme case (though for a sphere it does not make sense to set $\tau$ different to zero). The convexity strengthening is clearer in Figure 1 (right), where the method is applied to the mexican hat function. Cross sections of the recovered surface are shown, and from left to right they show the original surface and recovered surface after applying the method taking as a peak point the center of the surface with $\tau_{x}=\tau_{y}=0$. Note how the concave parts of the hat become convex.

In the following section some experiments will be presented in order to illustrate these points on an application involving face reconstruction using SFS.

\section{Experiments}

This section is organized into two parts. The first part describes experiments focussed on height recovery while the second part describes re-illumination tests.

The first series of tests were carried out on the image shown in Figure 2 (top-left)3. To compute the surface gradients from the raw image brightness we followed the procedure described in $[9]$. This construction ensures the image irradiance equation to be satisfied. For the surface integration step we used the global method proposed in [5] which recovers surface height using the inverse Fourier transform of the field of surface normal directions.
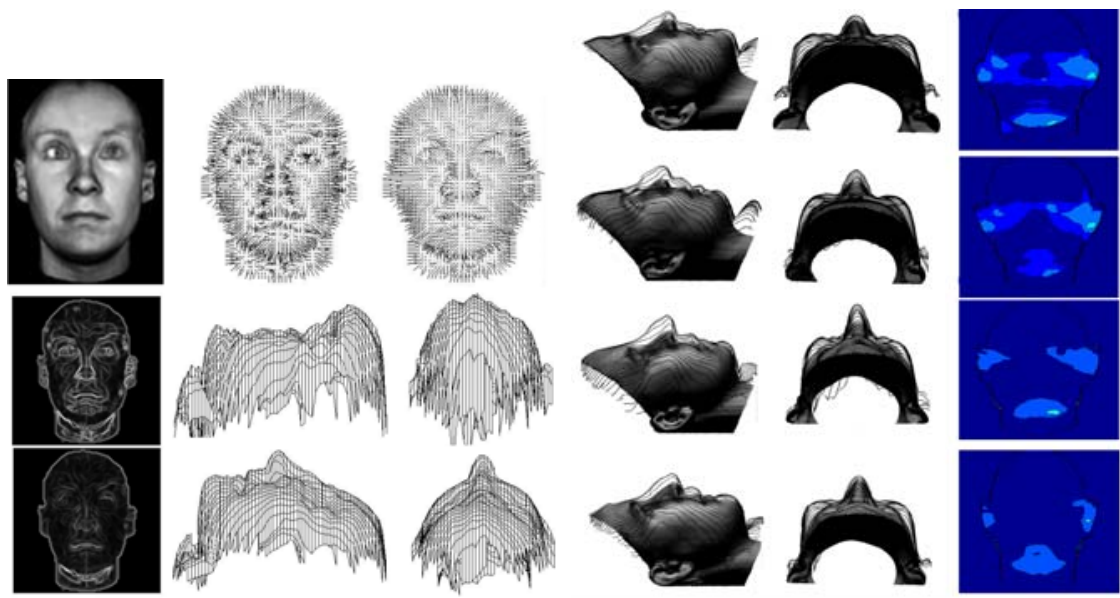

Fig. 2. Height and gradient analysis (see text)

In Figure 2 (three first columns), the first row shows the input image, the initial needle map and the curvedness-modified $\left(\tau_{x}=0.3\right.$ and $\left.\tau_{y}=0.4\right)$ needle map. It is worth mentioning that, for the hardest case, where no data is filtered by the thresholds, a four-quadrant effect is quite perceptible, but it tends to

${ }^{3}$ The face database was provided by the Max-Planck Institute for Biological Cybernetics in Tuebingen, Germany 
relax when the filter starts to work. The harder the threshold, the stronger the influence of the face features. On the other hand, the middle and bottom rows show, from left to right the curvedness map, together with profile and top-down views of the surface wire-frames recovered using surface integration. The next row is for $\tau_{x}=0.3$ and $\tau_{y}=0.4$, respectively. It is important to highlight how the change of signs in the surface normals suggests that the new derivatives arise now from the shape of a face (bottom row) rather than from the image of a face (middle row). By incrementing $\tau_{x}$ and $\tau_{y}$ the fine features of the face seem to be preserved and the overall structure of the face is still sound. By choosing an appropriate threshold we are able to enhance the salient features of a particular face while maintaining the overall face composition.

As far as the reconstruction using shape-index is concerned, the height data recovered is very similar to that obtained using curvedness. Figure 3 (left) shows a scatter plot comparison between the original and modified shape-indexes: the $\mathrm{x}$-axis corresponds to the original case while the $\mathrm{y}$-axis corresponds to the $\tau_{x}=$ $\tau_{y}=0.4$ case 5 . This diagram presents only those pixels in the original gradient field with a shape index lower or equal to 0.4 ( $x$-axis), therefore we can analyze the new value of such pixels in the redirected field of surface normals ( $y$-axis). Note how the majority of the points are distributed above the line $x=y$, which shows how the original shape index turned into a grater one, suggesting that the concave regions changed to convex. Such pixels belong to the regions surrounding the nose, mouth and eyes. The small cloud of points below the line, representing the pixels where the shape index remained lower than the threshold is mainly related to the pixels surrounding the face boundary.
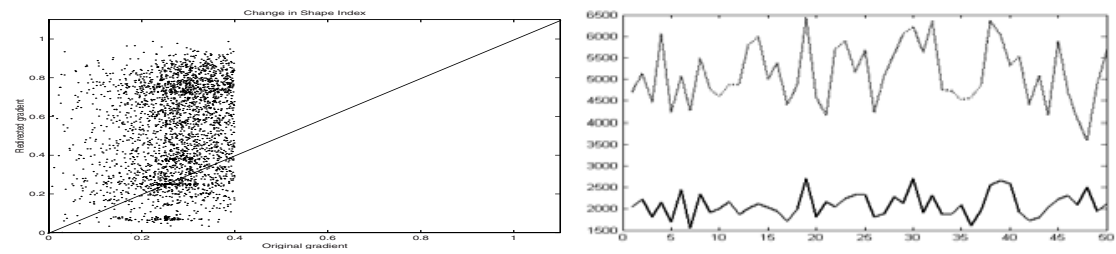

Fig. 3. Convexity enforcement using shape index and height difference tests (see text)

The images shown in Figure 2 (three last columns) provides an absolute height difference analysis. The left-most column shows the color-coded isocontour plot of the absolute height differences after re-directing the surface normals. The middle and right-most columns show superimposed plots of the recovered height maps after applying our method on the profile views of the images from which the original gradient fields were calculated. We can observe from the iso-contour images that the absolute height difference is diminished after changing the direction of the surface normals. Errors are most significant in the chin and eye areas. This might be a consequence of change of albedo (eye

\footnotetext{
${ }^{4}$ The shape-index scale was normalized from 0 to 1 .

${ }^{5}$ For all subsequent experiments, both $\tau_{x}$ and $\tau_{y}$ will slightly fluctuate between 0.3 and 0.4
} 
area) and instabilities produced by the boundaries of the chin and neck. From the superimposed, it is clear that the major differences in the recovered height maps and the ground-truth surfaces are near the nose, the cheeks and the mouth area.

To provide a more detailed analysis of our method, a more exhaustive set of tests was carried out on fifty images of faces from the database. The absolute height difference comparison plot 6 is shown in Figure 3 (right). The solid line shows the differences calculated on the surfaces integrated after using our method, while the dotted line shows the case of the un-altered gradients. It is obvious that after gradient re-direction the difference among the integrated surfaces decreases considerably, around $53 \%$ on average.
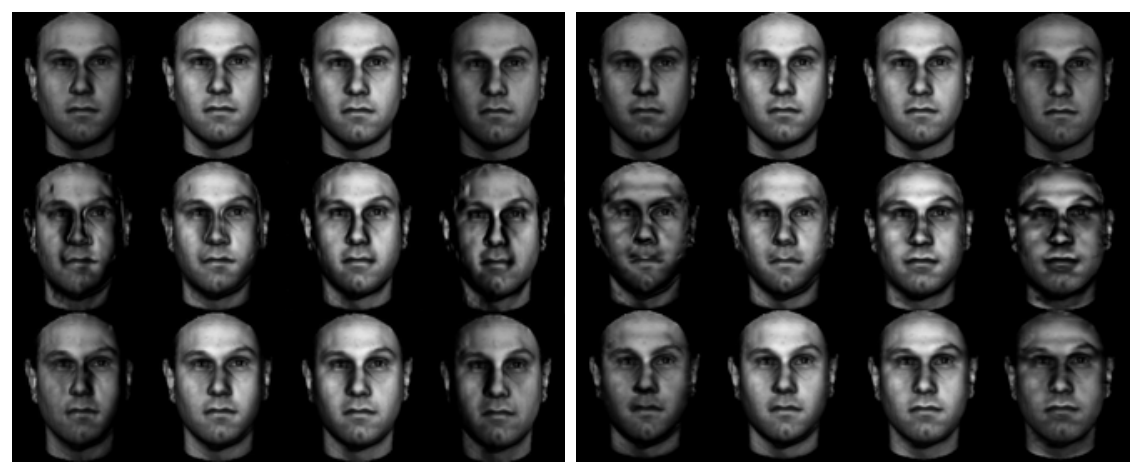

Fig. 4. Comparison for re-illumination tests (see text)

The following set of tests show how our proposed method for re-directing surface normals of faces can be used to produce differently illuminated versions of the face 7 . In Figure 4 we show some results of the re-illumination process. For both sets of images, the top row represents the re-illumination tests using the ground-truth normals, the middle row shows those obtained using the unaltered gradient field and the bottom row those obtained using the re-directed surface normals. From left to right, the light source direction is makes an angle of -45 , $-25,25$ and +45 degrees to the image normal in the horizontal (x) direction for the left-most set of images. In the right-most set of images, the light source is moved in the same manner in the vertical direction. It is interesting to note the similarities between the ground-truth and re-directed gradient re-illuminations. Although the recovered surface does not accurately represent the shape of the image from which it was acquired, the overall shape is good enough to create accurate re-illuminations provided that the light source is not moved by more that $45^{\circ}$. This results are best when the light source direction is moved in the

${ }^{6}$ For these experiments, the ground-truth surface was generated by integrating the known ground-truth gradient from each image, using the Frankot and Chellappa method. This was done so that all the surfaces were generated on the same basis for comparison purposes. This reduces the biases involved in the integration method.

7 The lambertian illumination model was applied, assuming as albedo maps the input images. 
horizontal direction, and this could be explained as a consequence of the vertical symmetry of human faces. On the other hand, the re-illumination results for the un-modified gradient fields show artifacts of implosion in the area around the nose and mouth. This becomes more severe when the light source moves further away from the viewer direction.

\section{Conclusions}

We have proposed an algorithm for correcting a gradient field of a face. The aims in doing this are twofold. First, we wish to generate a height map with a global maximum located at a critical point located at the tip of the nose. Second, we aim for force the recovered surface to be convex in accordance with evidence provided by local shape indicators. We have proved that the simple idea of modifying the surface normal directions so as to restore the convexity of imploded features using the constraints derived from the location of a point of global maximum height seems to work well with the recovery of face surfaces. After integration, the recovered shape seems to preserve most salient features, including the nose lips and eye-sockets. The constrain could be used for more general surfaces in a local manner for surface height recovery where there are local regions of implosion.

\section{References}

1. Atick, J., Griffin, P. and Redlich, N. (1996), Statistical Approach to Shape from Shading: Reconstruction of Three-Dimensional Face Surfaces from Single TwoDimensional Images, Neural Computation, Vol. 8, pp. 1321-1340.

2. Besl, P.J. and Jain, R.C. (1986) Invariant surface characteristics for 3-d object recognition in range images, Comput. Vision Graphics Image Proc., Vol. 33,pp. 33 -80 .

3. Dovgard, R. and Basri, R. (2004), Statistical symmetric shape from shading for 3D structure recovery of faces, European Conf. on Computer Vision (ECCV 04), Prague, May 2004.

4. Forsythe, D. and Ponce, J. (2001), Computer Vision: a Modern Approach, PrenticeHall.

5. Frankot, R.T. and Chellapa, R. (1988), A Method for Enforcing Integrability in Shape from Shading Algorithms, IEEE Trans. Pattern Analysis and Machine Intelligence, Vol. 10, No. 4, pp. 438 - 451.

6. Horn, B.K.P. and Brooks, M.J., (1989), Shape from Shading, MIT Press, Cambridge, $M A$.

7. Koenderink, J.J., and Van Doorn, A.J. (1992), Surface Shape and Curvature Scales, Image en Vision Computing, Vol. 10,pp. 557-565.

8. Prados, E. and Faugeras, O. (2004), A rigorous and realistic Shape From Shading method and some of its applications, INRIA Research Report, RR-5133, March 2004.

9. Worthington, P. L. and Hancock, E. R. (1999), New Constraints on Data-closeness and Needle Map Consistency for Shape-from-shading, IEEE Trans. on Pattern Analysis and Machine Intelligence, Vol. 21, No. 12, pp. 1250-1267. 